\title{
CORRESPONDENCE
}

\section{Comment on "Increased risk of second cancers at sites associated with HPV after a prior HPV-associated malignancy, a systematic review and meta-analysis"}

\author{
British Journal of Cancer (2019) 120:954-955; \\ https://doi.org/10.1038/s41416-019-0437-2
}

The systematic review and meta-analysis study "Increased risk of second cancers at sites associated with HPV after a prior HPVassociated malignancy, a systematic review and meta-analysis", presented by Gilbert et al. and published in BJC, has been read by us with interest. ${ }^{1}$ Our meta-analysis research team would like to bring into attention a few valid improvements to the paper. Therefore, this study may serve a better position as a citable article.

\section{SMALL SAMPLE SIZE AND WIDER CONFIDENCE INTERVAL FOR QUANTITATIVE SYNTHESIS}

A question that presents itself upon viewing the data presented in the study is the choice of Gilbert et al. to conduct a meta-analysis when a number of included studies have very small sample sizes. The problems that arise upon choosing to include studies with small sample sizes is self-evident in Figure 3 of Gilbert et al.'s paper, where we observed that the number of studies (the studies in question have been listed in the Table 1 given below) have very wide percent of Confidence Intervals $(\mathrm{Cl})-95 \% \mathrm{Cl}$, going beyond the limits of the constructed Forest Plot. Wide Cl's indicate a lack of precision, and the presence of the high degree of standard error, which in turn is a characteristic symptom of a having a small

Table 1. A number of included studies have wide $95 \%$ confidence intervals $(\mathrm{Cl})$ in the forest plot

\begin{tabular}{lll}
\hline Author & Second primary cancer type & Number of cases \\
\hline Fisher et al. & Cervical & 5 \\
Mitchell et al. & Cervical & 6 \\
Neumann et al. & Vulvo-vaginal & 8 \\
& Anal & 5 \\
& Oropharynx & 2 \\
Hemminki et al. & Cervical & 7 \\
& Anal & 1 \\
& Oropharynx & 2 \\
Saleem et al. & Anal & 5 \\
Frisch et al. & Cervical & 2 \\
& Vulvo-vaginal & 5
\end{tabular}

sample size. ${ }^{2,3}$ This issue needs to be addressed as a major limitation of this study.

\section{INDIVIDUAL STUDY WEIGHT AGAINST POOLED ESTIMATED EFFECT SIZE}

Furthermore, the meta-analysis conducted does not include the mention of the individual weights of the study, which leads to further uncertainty, as readers are unable to clearly ascertain the degree of impact that the small sample size studies have upon the overall pooled Standardised Incidence Ratios (SIRs). ${ }^{4}$

\section{IS SIRS INTERCHANGEABLE WITH HAZARD RATIO (HRS)?}

The abovementioned issues are exacerbated when we observe that the authors have used the term SIRs interchangeably with HRs, particularly in the forest plots (Figure 2 and Figure 3) where they continue to describe the SIRs in the legends of each figure, while the figures themselves seem to represent HRs. The estimated or pooled effect size of the risk of HPV-associated cancer after prior diagnosis is SIR in this meta-analysis. In statistical terms, HRs are markedly different from SIRs, as the former denotes hazard rates corresponding to the conditions described by two levels of an explanatory variable, while the latter describes the ratio of the observed number of cases to the expected number of cases, and therefore, must not be used interchangeably. ${ }^{5}$

\section{PUBLICATION BIAS IS A CRUCIAL INDICATOR OF META- ANALYSIS IN CLINICAL RESEARCH}

It is also significant that the study did not evaluate the presence of publication bias in its included studies. Despite its absence being a legitimate threat to the validity of any meta-analysis study, few meta-analysis studies include it in their analysis, even though methods to assess it has been addressed in previous studies, elaborating on its importance. ${ }^{6-8}$

\section{ESTIMATED VARIATION OF HETEROGENEITY BETWEEN STUDIES}

Another point of consideration would be the test of betweenstudy heterogeneity. The authors have used the Higgins I-squared statistic to analyse the meta-analysis data. However, the I-squared statistic may not be sufficiently informative as it does not consider the threshold effect. We want to suggest that Kendall's Tausquared statistic to be included. This Tau-squared statistic serves as the estimated variation of heterogeneity between the effects for test-accuracy observed between studies, while also considering the threshold effect. ${ }^{9}$

These issues are not only relevant to Gilbert et al.'s study, but also for all systematic reviews and meta-analysis which may seek to follow up on similar lines of study. 


\section{ADDITIONAL INFORMATION}

Competing interests: The authors declare no competing interests.

Note: This work is published under the standard license to publish agreement. After 12 months the work will become freely available and the license terms will switch to a Creative Commons Attribution 4.0 International (CC BY 4.0).

Publisher's note: Springer Nature remains neutral with regard to jurisdictional claims in published maps and institutional affiliations.

Rama Jayaraj ${ }^{1}$ and Chellan Kumarasamy ${ }^{2}$ ${ }^{1}$ Clinical Sciences, College of Health and Human Sciences, Charles Darwin University, Ellengowan Drive, Casuarina, NT 0909, Australia and ${ }^{2}$ University of Adelaide, North Terrace Campus, Adelaide, SA 5005, Australia Correspondence: Rama Jayaraj (Rama.Jayaraj@cdu.edu.au)

\section{REFERENCES}

1. Gilbert, D. C., Wakeham, K., Langley, R. E., Vale, C. L. Increased risk of second cancers at sites associated with HPV after a prior HPV-associated malignancy, a systematic review and meta-analysis. Br. J. Cancer 120, 256-268 (2018).
2. du Prel, J.-B., Hommel, G., Röhrig, B. \& Blettner, M. Confidence interval or p-value?: part 4 of a series on evaluation of scientific publications. Dtsch. Arztebl. Int. 106, 335-339 (2009).

3. Hazra, A. Using the confidence interval confidently. J. Thorac. Dis. 9, 4125-4130 (2017).

4. Bown, M. J. \& Sutton, A. J. Quality control in systematic reviews and meta-analyses. Eur. J. Vasc. Endovasc. Surg. 40, 669-677 (2010).

5. Stare, J. \& Maucort-Boulch, D. Odds ratio, hazard ratio and relative risk. Metodo. Zv. 13, 59-67 (2016).

6. Jayaraj, R., Kumarasamy, C., Ramalingam, S., Devi, A. Systematic review and metaanalysis of risk-reductive dental strategies for medication related osteonecrosis of the jaw among cancer patients: approaches and strategies. Oral Oncol. 86, 312-313 (2018).

7. Jayaraj, R., Kumarasamy, C., Sabarimurugan, S. \& Baxi, S. Letter to the Editor in response to the article," The epidemiology of oral human papillomavirus infection in healthy populations: a systematic review and meta-analysis". Oral. Oncol. 84, 121-122 (2018).

8. Kumarasamy, C., Devi, A. \& Jayaraj, R. Prognostic value of microRNAs in head and neck cancers: a systematic review and meta-analysis protocol. Syst. Rev. 7, 150 (2018).

9. Sabarimurugan, S., Madurantakam Royam, M., Das, A., Das, S., K, M. G. \& Jayaraj, R Systematic review and meta-analysis of the prognostic significance of miRNAs in melanoma patients. Mol. Diagn. Ther. 22, 653-669 (2018). 